\title{
Implementation of Tea Harvester
}

\author{
Manikanta M, Niranjan R Sansuddi, \\ Neha Shetty, Yashas K B \\ Department of Electrical and Electronics Engineering \\ R V College of Engineering \\ Bangalore, India
}

\begin{abstract}
Tea is one of the most commonly used beverages across the world. India is the second largest tea producer in the world. Over 70 percent of tea leaves are consumed within India. The tea leaves are harvested by different methods. The conventional methods have certain disadvantages like overweight, pollution, low yield and high cost of machine. This paper includes design to overcome the limitations of conventional machine. The mechanical design of the harvester involving roller, blades, conveyor mechanism, height adjustment, wheels, storage. The electrical connections involving controllers, sensor, drivers is done using Proteus design suite. The integration of the mechanical designs with electrical connections makes the harvester complete. The designed and developed model is suitable to harvest tea leaves with gradient of 5 percent. The novelty of this model involves addition of sprinkler to the harvester. This makes work much easier and smarter. The harvester is remotely controlled by the user through the application developed. This completely makes the machine more durable and reliable for harvesting tea leaves.
\end{abstract}

Keywords - Tea plucking; harvester; tea harvester; sprinkler;

\section{INTRODUCTION}

Tea is one of the foremost popular and healthy beverages around the world. India stands to be the second largest tea producer in the world [1]. India is also a largest consumer of tea as well. The favoured tea beverage in India is the Assam tea and also the Darjeeling Tea. The Indian tea industry has grown to have many global tea brands and has evolved into one of the foremost technologically equipped tea industries within the world. Tea is manufactured by processing the leaves within the factories that are plucked and graded with various grades counting on the standard. A milestone in the tea industry is during 1990-91, India implemented a policy called Liberalization, Privatization, and Globalization (LPG), it created a harsh competition between firms and affected the tea industry also [2]. The tea industry in India has suffered in some ways like lowering of tariff barriers, fewer limits on imports, shortage of labourers, decrease in wages and attack of pests. The tea leaves are usually harvested in many ways. The conventional methods are hand plucking, diesel operated machines, and petrol operated machines, highly automated machines. The main drawbacks of such machines are as follows; they weigh up to 18 kilograms so it requires more labour for the work. The smoke outputs from the machine make the leaves to shed soon, also it affects the health of the labour [4]. The costs of machines are usually high. These key factors have made the engineers mind to design a harvester. The study showed the harvester used in recent years have drawbacks like pollution, requires more labour, bulky machines etc.

To overcome these hindrances as discussed, a design is proposed having fewer limitations than the prevailing

\author{
Dr. Hemalatha J N \\ Department of Electrical and Electronics Engineering \\ R V College of Engineering \\ Bangalore, India
}

designs. The proposed semi-automatic system has a roller with blade arrangement that harvests the tea leaves automatically and is integrated with a pesticide sprayer; it doesn't compromise on crop safety and therefore the production rate. This tea harvesting machine is more viable, feasible, lighter in weight and profitable than manual harvesting [5-8]. The proposed model is originally designed to be a working porotype model but had to be developed entirely on software platform using solid works and Proteus design suite for validation due to the corona virus crisis. The model is tested for its working in the Proteus software by developing the circuit in Proteus and running it for various simulations [9-10]. The working of the various functions such as the movement, plucking, collection of leaves etc. are all tested and validated using the Proteus software.

An android application is developed to control all the functions and a Bluetooth module is provided in the Proteus circuit for integration. The model is designed on solid works for the movement of the tea harvester and also the plucking mechanism is made visible in the animation of the model. Various functions such as plucking, the working of the pesticide sprayer, conveyor belt is also seen in the animation model.

In this paper section II discusses the block diagram of harvester, followed by the Development of 3D model with Android Application to control the harvester. The simulation study of harvester is done on Proteus Design suite.

\section{BLOCK DIAGRAM OF THE PROPOSED TEA HARVESTER}

The block diagram of proposed tea harvester is shown in Figure 1.

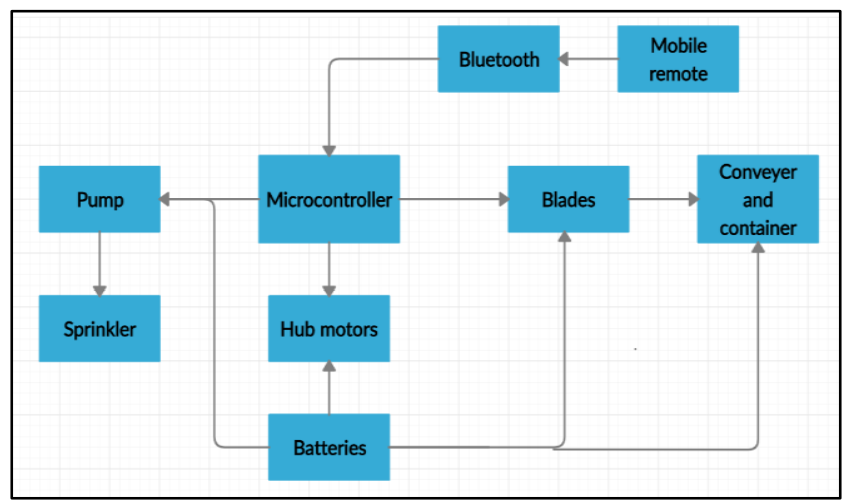

Fig 1. Block Diagram of proposed Tea Harvester

The tea harvester is controlled with help of an Android application. The application acts as a client and harvester as a 
server. The connectivity is through Bluetooth. The app is provided with functionalities to control the harvester in all aspects. The command from the client is first received by the Bluetooth module $\mathrm{HC}-05$ and the signals are sent to controller. The controller adopted here is Arduino Uno and Nano. Controller is programmed to control the motor of all the mechanism.

In the front, the blades serve the purpose of cutting tea leaves. It does its function with help of roller. The conveyor mechanism is to stack the tea leaves plucked by the blade mechanism and to place it to storage unit. The power to all the electrical and electronic components is powered by the Battery and is places under the conveyor.

Behind the harvester, the tea leaves are sprinkled with nutrients after the machine is passing ahead. To pump the nutrients from the nutrient tank a pump is placed. The HUB or in wheel motors are housed inside the wheels to provide movement of harvester. The machine is not provided with chassis for turning effect because of design and application constraint.

\section{MODELLING OF PROPOSED TEA HARVESTER}

The harvester model is first sketched in two dimension and later it was done in three dimension using solid works software.

\section{A) Two-Dimensional Model of Tea Harvester}

The proposed sketch of the tea harvester model and its CAED design is as shown in Figure 2, Figure 3 and Figure 4 respectively.

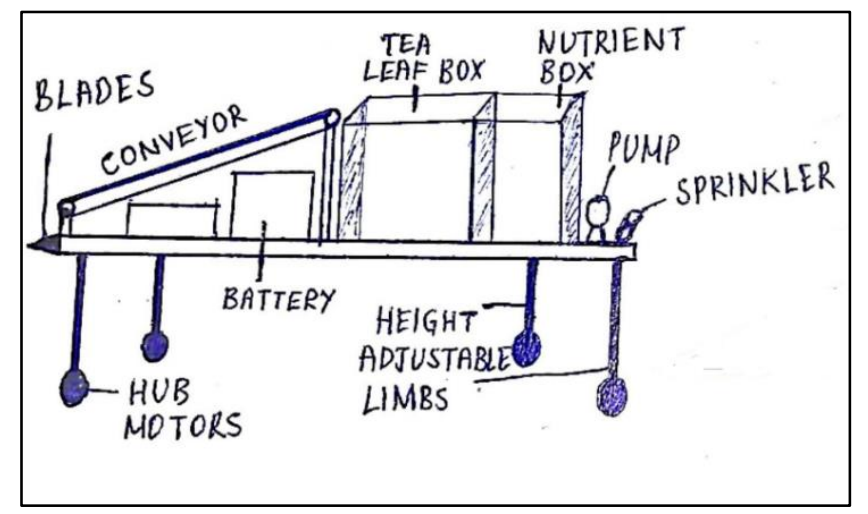

Figure 2. Proposed Sketch Side View

All the dimesnion are in $\mathrm{mm}$. The side view and top view of the model is displayed. The design proposed includes all the functionalities of the Tea Harvester. The dimension of the mechanism is shown right side figure2. The developed model using CAED is shown in figure 3 and figure 4.
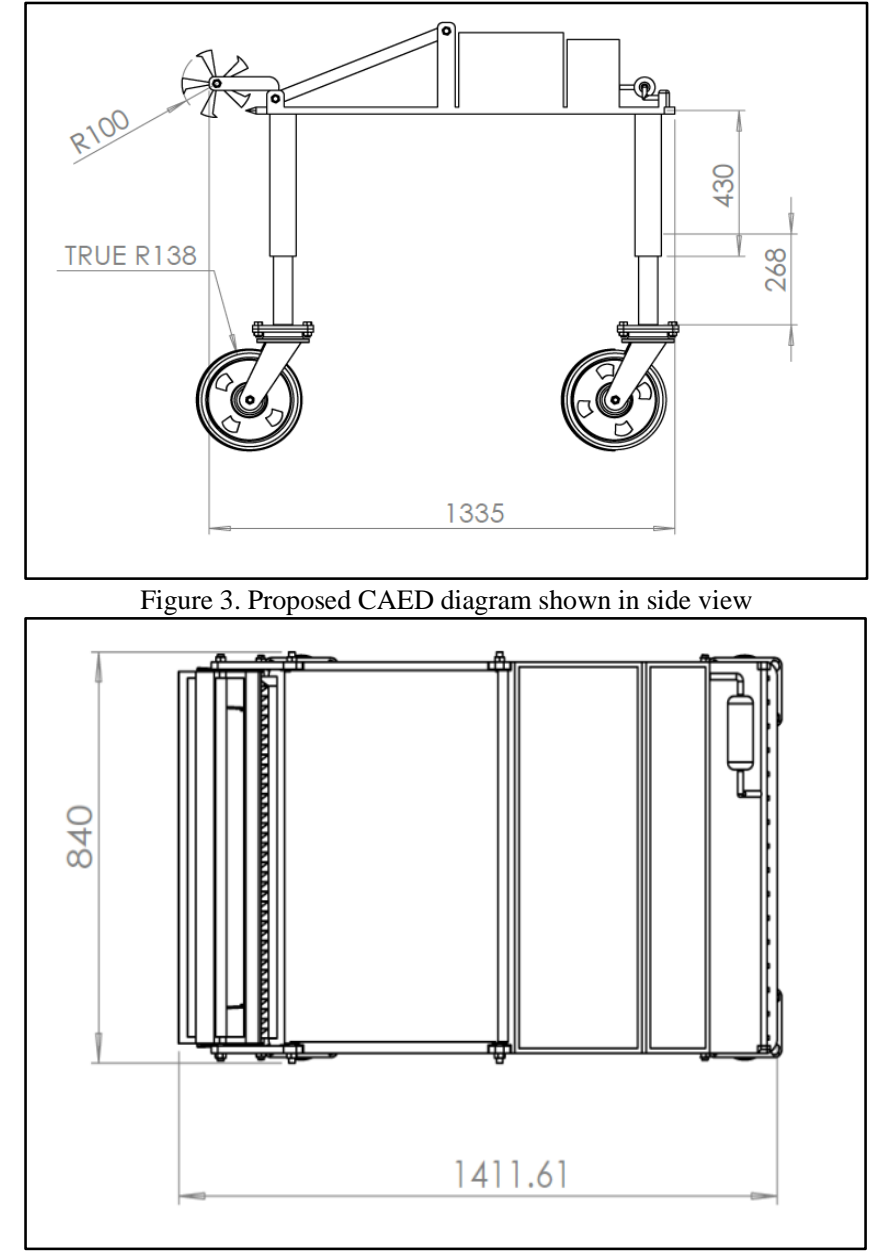

Figure 4. Proposed CAED diagram shown in Top View.

The dimension of the model developed in the CAED software is in mm. Figure 4 shows the Top view of harvester developed using CAED.

\section{B) Three-Dimensional Model of Three Harvester}

The three-dimensional design of the Tea Harvester is done using the software called Solid works. The mechanism of the tea harvester is developed by designing individual parts. The parts are initially developed in 2 Dimension and then extruded to get the 3 Dimensional models. All the parts are later assembled in the assembly section of Solid works. Once the model is developed, motion is inserted to individual mechanism. All the motion are adhered to design and application constraints.

The 3 Dimensional models consists of the following parts

$>\quad$ Leaf Plucking Unit

$>$ Roller and Blade Mechanism

$>$ Leaf Collection Unit

$>$ Conveyor Belt Mechanism

$>$ Battery Box Storage Unit

$>\quad$ Nutrient and Tea Leaves Storage Unit

$>$ Sprinkler Mechanism

$>$ Height Adjustable Limb Mechanism

$>$ Wheel Mechanism 
- Leaf Plucking Unit:

The leaf plucking unit consists of a roller made of six bars and moves in a circular motion. In its motion, the roller first supports the leaf and the two layers of blades moving in a to and fro motion cut the tea leaf stems. After it is cut, the roller moves the tea leaves onto the conveyer belt that transforms it into the leaf collecting unit.

- Roller and Blade Mechanism:

The roller is designed in such way that it acts as an alternative for plucking mechanism. It consists of six bars for supporting tea leaves and the supported leaves are cut by the blades. The roller blade mechanism developed in the solid works platform is shown in figure 5 .

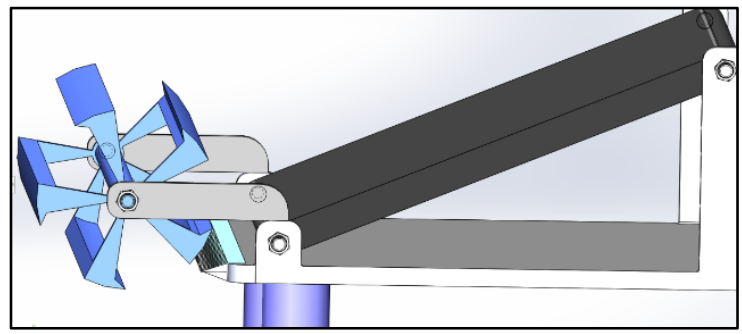

Figure 5. 3D views of Roller and Blade Mechanism.

- $\quad$ Leaf Collection Unit:

The leaf collection unit consists of the conveyer belt and a box unit on the platform for collecting the tea leaves. The leaf after being cut is transported from the cutting unit to the collection unit in a conveyer belt to be stored. A door is also provided for easy collection of the tea leaves after the box is filled

\section{- Conveyor Belt Mechanism}

The tea leaves after it is cut by the roller and blade mechanism is made to enter conveyor mechanism. The conveyor has driver and driven pulley. The speed of the conveyor is made slow so that the tea leaves get stacked and then shifted to tea storage unit. The conveyor belt mechanism is shown in figure 6 .

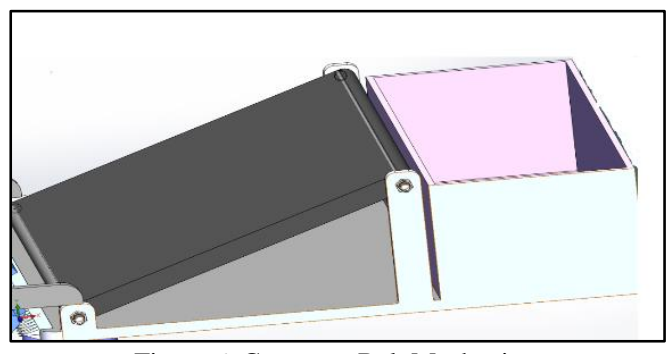

Figure 6. Conveyor Belt Mechanism

- Battery Box Storage Unit :

The design of the tea harvester includes storage for the batteries to power the tea harvester. The optimum location of the batteries to power its electrical components such as the hub motors is near the cutting unit and the front wheels and hence the battery box is located closer to the front portion. In the battery box, the batteries, the boost converter circuit and the controller are located.

- $\quad$ Nutrients And Tea Leaves Storage Unit :
The pesticides like Flubendiamide and Emamectin Benzoate and nutrients/minerals has to be sprayed soon after the harvesting of the tea leaves that increases the production of tea leaves for the next yield. A separate nutrient box is also provided on the platform and is used to store the nutrients that are sprinkled on the tea leaves right after plucking. Spraying of nutrients helps in the healthy growth of new tea leaves. The nutrients and tea leaves storage unit is as shown in figure 7.

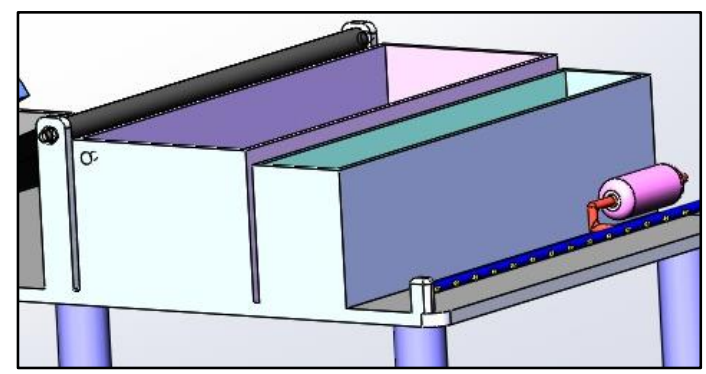

Figure 7. Nutrients and Tea Leaves Storage Unit

- Sprinkler Mechanism:

The sprinkler mechanism is added to this model so as to sprinkle the nutrients on to the bush right after chopping the leaves. This is done to increase the yield. Figure 8 shows Sprinkler Mechanism.

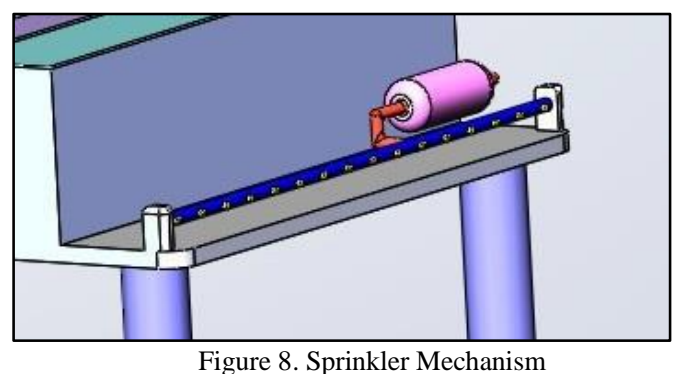

- Height Adjustable Limb Mechanism :

The tea plantation is not plain in hilly regions. In order to compensate the height of machine a height adjusted mechanism is provided in the design. The height adjustment is provided with lead screw mechanism. The designed limb model developed using the Solid works is shown in figure9.

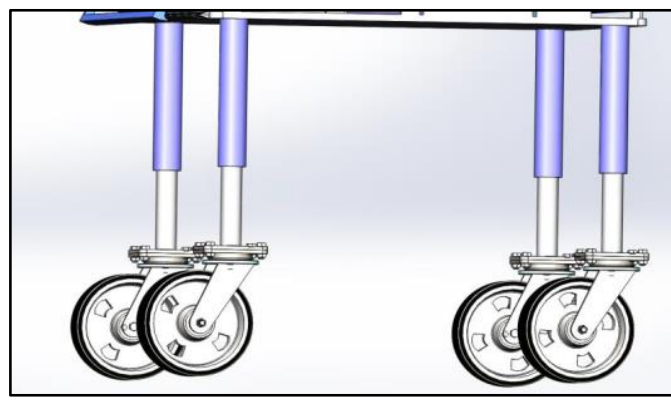

Figure 9. Height Adjustment Mechanism.

- Wheel Mechanism:

The wheel mechanism is for movement of tea harvester across and along the field. The wheel is provided with degree freedom of 120 degree for changing the direction 
of harvester. The wheel is provided an animation for forward, reverse and left and right direction. The wheel mechanism is as shown in Figure 10.

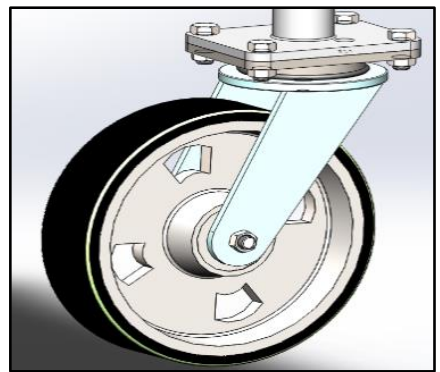

Figure 10. Wheel Mechanisms.

The integrated view is obtained by integrating all the parts together as shown in Figure 11 a and Figure11 b. The figure 11a shows the harvester in right side view with all the mechanism labelled.

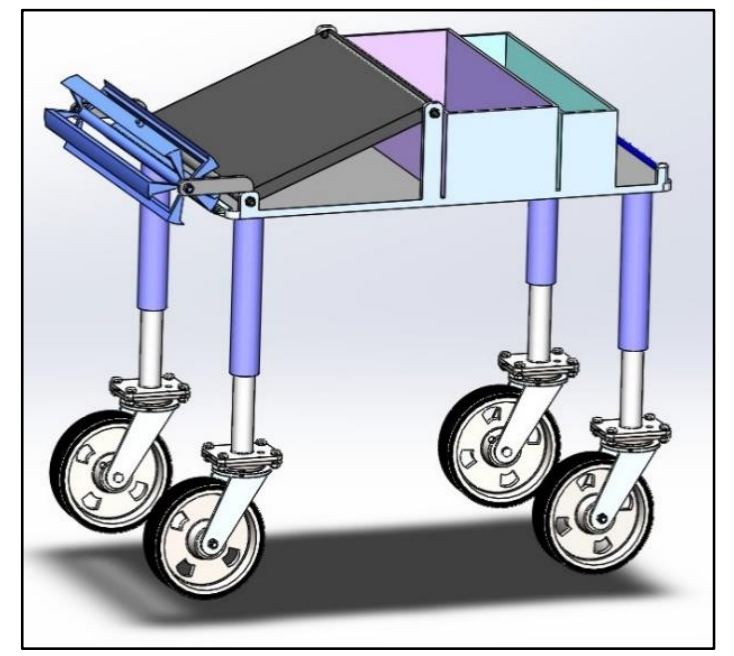

Figure 11 a. Side view of Tea Harvester developed in Solid Works

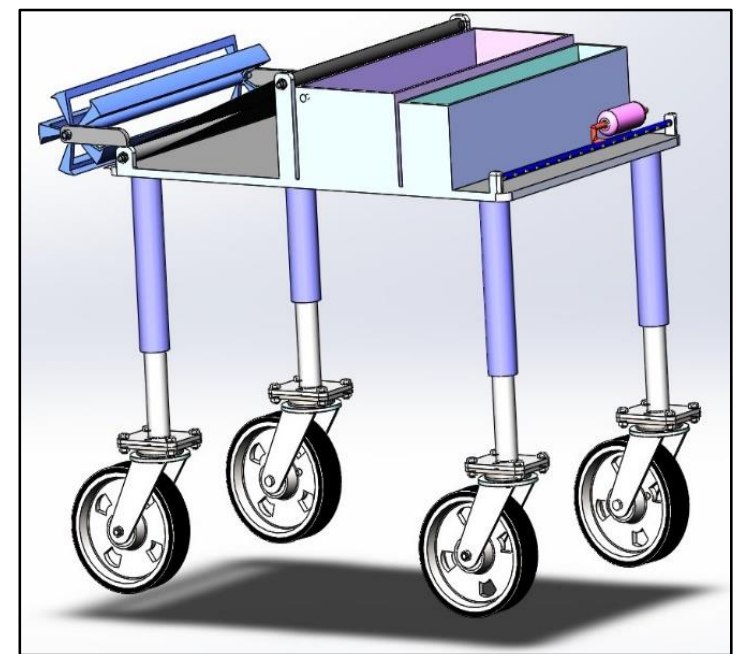

Figure 11 b. Back View of Tea Harvester developed in Solid Works

\section{C) Design of the App for Control of Tea Harvester}

The proposed tea harvester has several functions such as sprinkling the nutrients, steering of the vehicle, height adjustment and the conveyor mechanism; all these are controlled remotely via the developed Android Application. The developed application works based on Bluetooth Connectivity 4.0. This android application has been developed on MIT App developer platform. This platform uses graphical user interface that allows the users to drag and drop visual objects to create an application. There are 2 sections on the MIT app developer platform to develop the application and they are: designer and the blocks. In the designer section it is able to edit the appearance of the app and able to add visible elements such as the buttons, list pickers. Non visible elements such as the Wi-Fi and Bluetooth server and clients are also added. The function of each button and its logic is defined in the block section. The figure 12 shows snapshot of Android App developed.

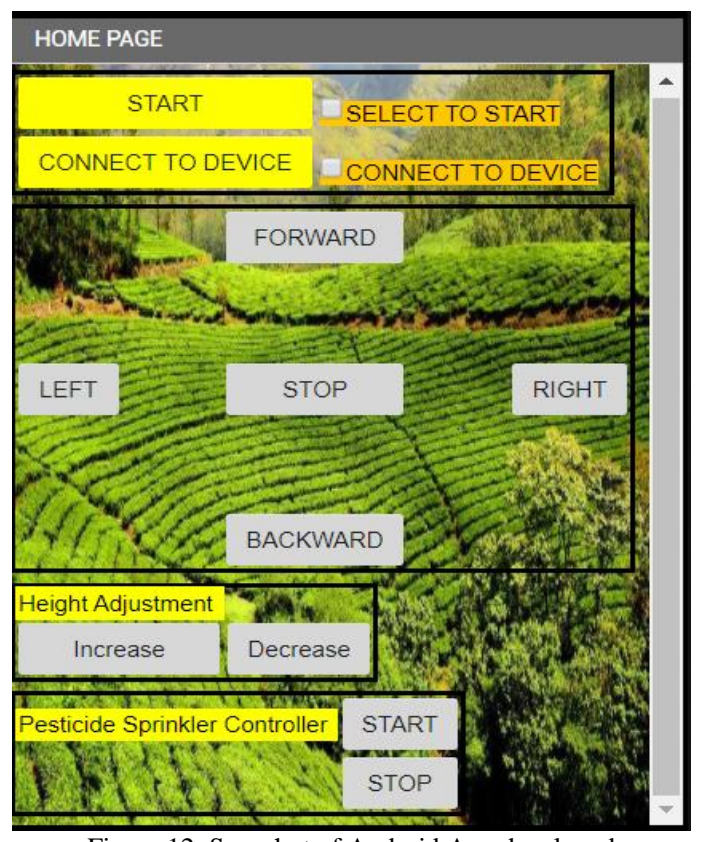

Figure 12. Snapshot of Android App developed

\section{SIMULATION STUDY ON PROTEUS DESIGN SUITE}

The circuit diagram to control the motor for various applications is shown in Figure 13. As explained in the previous section the circuit involves 3 micro controllers for controlling the actions of tea harvester, 4 motors for roller, SS blades, conveyor and Pump respectively. 2 motors for movement of vehicle or harvester in all the 8 directions. 4 motors are for height adjustment of limbs. All motors are driven by a driver circuit called L293D. 


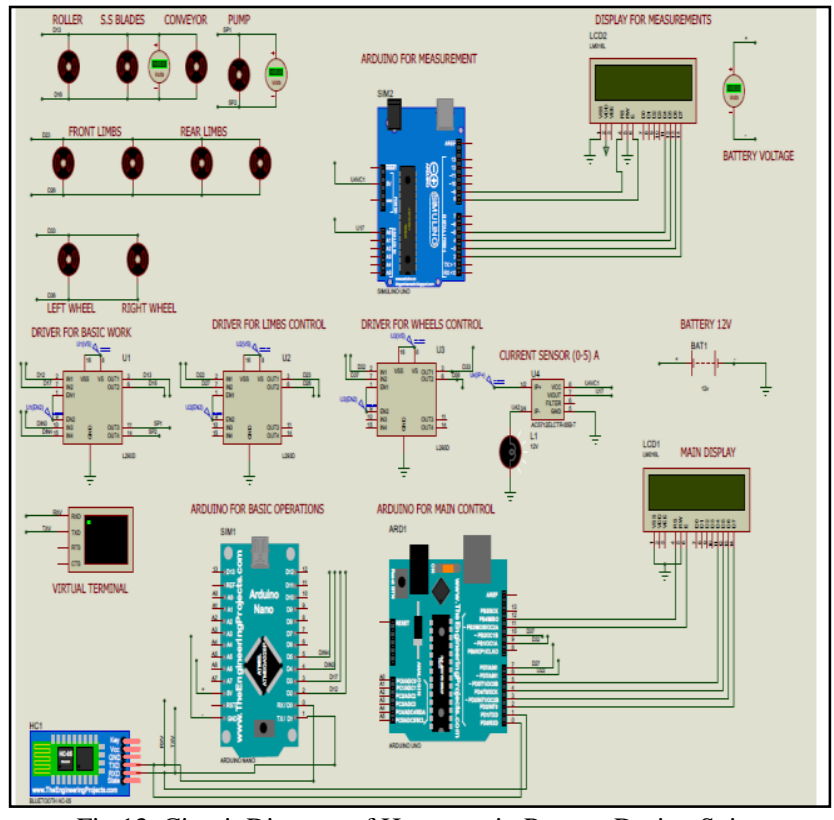

Fig 13. Circuit Diagram of Harvester in Proteus Design Suite.

\section{RESULTS}

The simulation study on Proteus Design suite is divided into three sections as follows

- Vehicle Movement.

- Height Adjustment.

- Measurement of parameters.

Case 1: Vehicle Movement

The Harvester is dependent on wheel for it's to and for movement. The harvester has three sets of speed ranges. The controller is programmed for 3 different speeds in scale of 10 RPM, 20 RPM and 30 RPM. As the user selects 1 from the app, the wheels run at a speed of 10RPM, selected 2 , wheels run at 20 RPM and if 3 is selected the wheel run at 30 RPM. This is done by PWM technique. The snap shots of wheels running at 10 RPM and voltage across the wheels is shown in Figure 14.a.

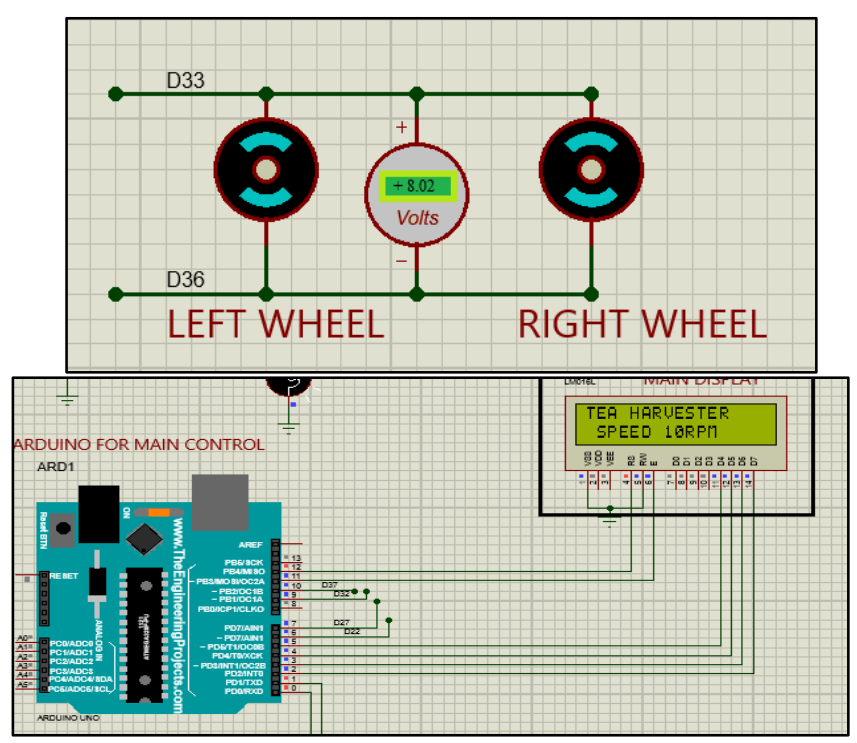

Figure 14.a. Wheels running at 10 RPM

The wheels running at 20 RPM and the voltage across the wheels at 20 RPM are shown in 14.b and 14.c respectively.

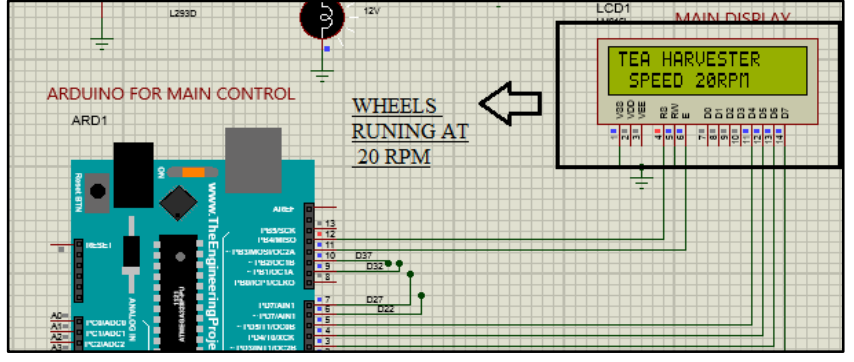

Figure 14.b. wheels running at 20RPM

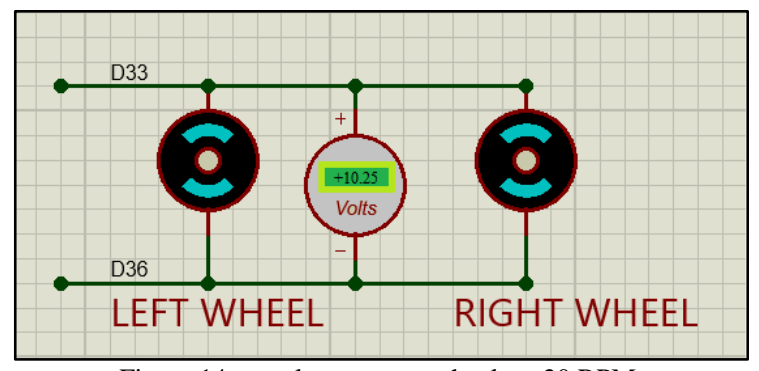

Figure 14.c. voltage across wheels at 20 RPM.

The wheels running at 30 RPM and the voltage across the wheels at 20 RPM are shown in 14.d and 14 e respectively.

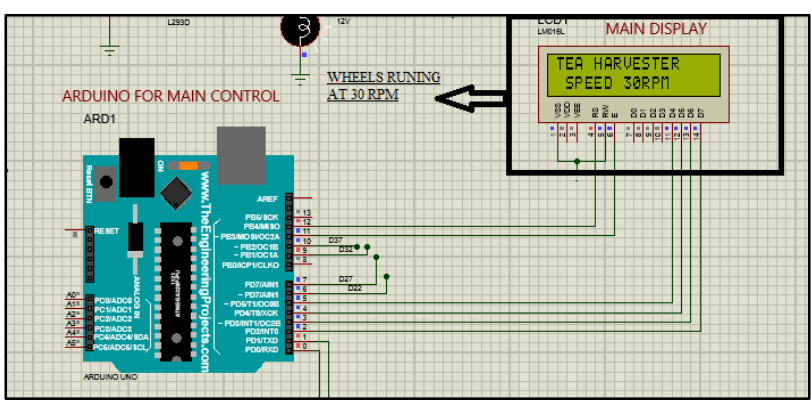

Figure 14.d. wheels running at 30RPM

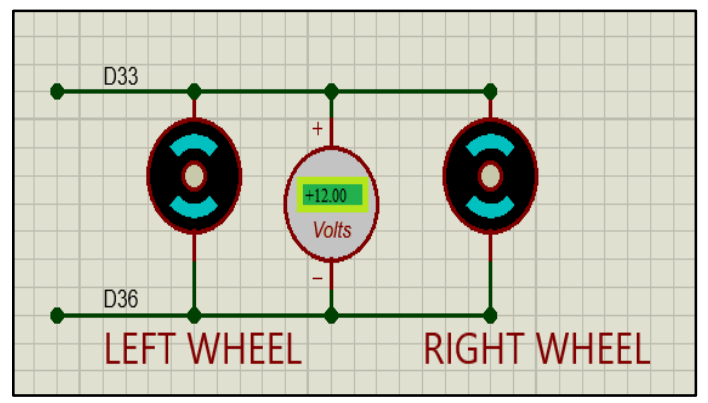

Figure 14.e. voltage across wheels at 30 RPM.

Case 2 : Height adjustment

The height adjustment of Limbs is done either automatically or manually. The command given through the application is displayed on LCD. The height of the harvester is adjusted from low to high and vice versa. This control is initialised with the app developed. The stroke of $200 \mathrm{~mm}$ is provided. The limbs move from $0 \mathrm{~mm}$ to $200 \mathrm{~mm}$ with a time lapse of 1.5 seconds. An option is provided to user if limb has to be interrupted in between $200 \mathrm{~mm}$. A snapshot of this operation is shown in figure 15 . 


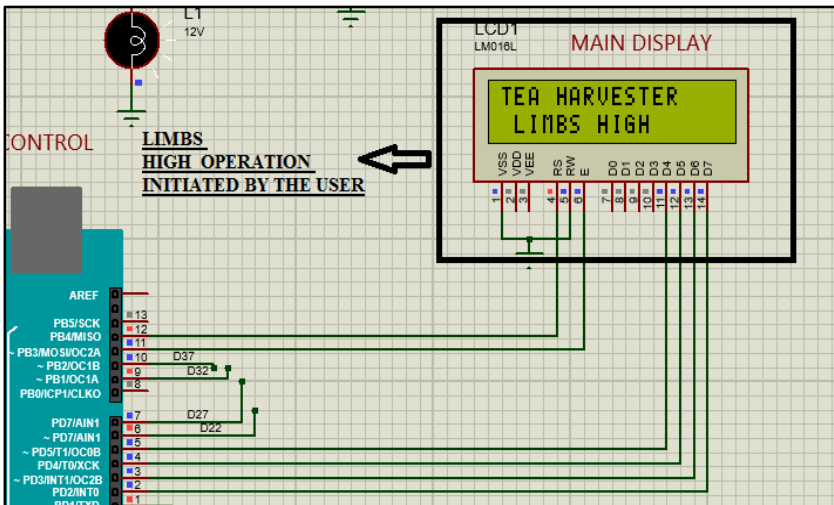

Figure 15. Snapshot of Limbs High operation.

The limbs move from $200 \mathrm{~mm}$ to $0 \mathrm{~mm}$ with a time lapse of 1.5 seconds. An option is provided to user if limb has to be interrupted in between $200 \mathrm{~mm}$. A snapshot of this operation is shown in Figure 15.a.

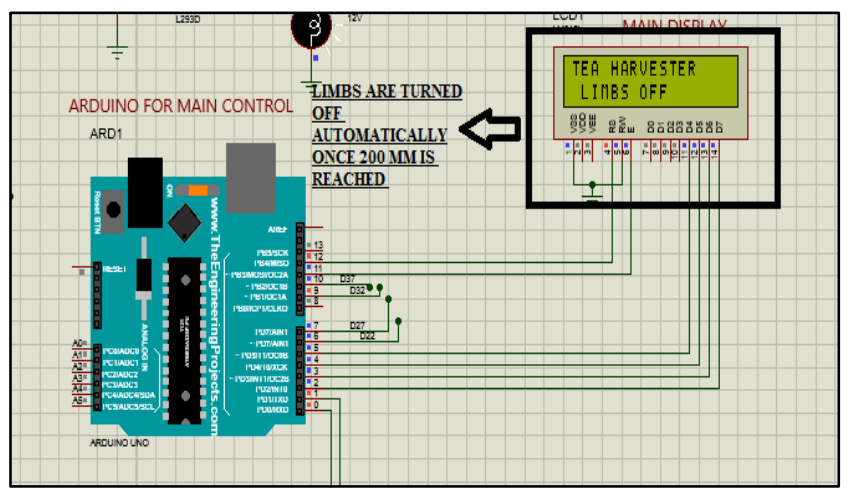

Figure 15.a. Snapshot of Limbs OFF operations.

- Measurement of parameters

The parameters like battery voltage, current, conveyor voltage, pump voltage are monitored and tabulated in the table form. This is to ensure that all the motors receive full voltage across them to function perfectly. The conveyor motor is slowed because to stack the leaves once they are cut by the roller and blades. The voltage of different motor is tabulated in table 1 .

\begin{tabular}{|l|l|l|l|}
\hline Sl.no & Device & Voltage & Current \\
\hline 1 & Conveyor & 7.93 volts & 0.78 Amps \\
\hline 2 & Pump & 9.89 volts & 0.36 amps \\
\hline 3 & Wheels & 12 volts & 1.26 amps \\
\hline 4 & Limbs & 8 volts & 1.02 amps \\
\hline 5 & Battery & 12 volts & 3.5 amps \\
\hline \multicolumn{4}{|l}{ Table 1. Voltage Across Different motors }
\end{tabular}

\section{CONCLUSION}

The proposed tea harvester with its multiple functions is able to overcome many hindrances compared to the previous methods of harvesting. The labour force required also reduces and the yield of the harvest increases. The results of the software validated the intended design and all the working parts of the machine were checked for its movement. The bandwidth of the Bluetooth app is about 5 meters. The model developed is able to store Tea Leaves weighing up to 10 kilograms. The sprinkler storage capacity is 1.5 litres. The height adjustment is provided for a stroke length of 0$200 \mathrm{~mm}$. The developed model was simulated for different speeds of 10, 20 and 30 RPM. The results of the software validated the intended design and all the working parts of the machine were checked for its animated movement in solid works.

\section{REFERENCES}

[1] Yu Han, Hongru Xiao, Guangming Qin, Zhiyu Song and Wenqin Ding, Song Mei, "Developing Situations of Tea Plucking Machine", Research Institute for Agricultural Mechanization, Ministry of Agriculture, Nanjing, China, Scientific Research Publishing Inc. 2014.

[2] Shambhavi Shankar, A.S.Ragool Delcien , Sumithra.C.Shenoy and Soumya S.H ,Anupama, "Semi-automatic Tea Leaf Harvesting Machine", International Journal of Advanced Research in Electronics and Communication Engineering, Volume 5, Issue 4, April 2016.

[3] Sureshkumar.A, S.Muruganand, Azha Periasamy and K. Sriram, P. Balakrishnan, "Design and Development of Selective Tea Leaf Plucking Robot", National Conference on Recent Trends in Electronics and Instrumentation 2013.

[4] Senthil Kumar Thangavel and Manesh Murthi, "A Semi Automated System for Smart Harvesting Of Tea Leaves", International Conference on Advanced Computing and Communication Systems (ICACCS -2017), Jan. 06 - 07, 2017, Coimbatore, INDIA.

[5] Ramaswamy Ravichandran and Parthiban Ramaswamy, "The impact of mechanization of tea harvesting on the quality of south Indian CTC teas", Elsevier Journal Food Chemistry, Vol. 63, No. 1, pp. 61-64, 199801998 Elsevier Science Ltd.

[6] Hai Vu, Thi-Lan Le, Thanh-Hai Tran and Thuy Thi Nguyen, "A Vision-Based Method for Automatizing Tea Shoots Detection", IEEE International Conference on Image Processing 2013.

[7] P. J. Burgess, M. K. V. Carr, F. C. S. Mizambwa, D. J. Nixon, J. Lugusi and E. I. Kimambo, "Evaluation of Simple Hand-held Mechanical Systems for Harvesting Tea”, expl agric. (2006), volume 42, pp. 165-187 2006 Cambridge University Press.

[8] Chia-Chang Wu, "Developing Situation of Tea Harvesting Machines in Taiwan", Engineering, Technology \& Applied Science Research, Tea Research and Extension Station Taoyuan, Taiwan, R.O.C. 2015.

[9] R.K.P.S. Ranaweera , H.K.G. Punchihewa , L.N.S. Wijayasingha, K.P.P. Sanjeeva and C.M. Samarakoon, "Selection of Harvestable Tea Shoots based on Height and Stiffness", Conference paper 1st Manufacturing \& Industrial Engineering Symposium (MIES 2016) At: Colombo, Sri Lanka.

[10] Shubham Kumar Jain, Anubhav Jain, Aditya Goswami, Rajesh Purohit, Anantha Sai Hari Haran Vinjarapu and Suresh Kumar Gawre, "IoT based Smart Tea leaves Plucker with Two Revolute type Planar Manipulator", 2018 IEEE International Students' Conference on Electrical, Electronics and Computer Science. 